\title{
Meaningful use of electronic health record (EHR) medication reconciliation technology requires a shared understanding of its value among healthcare providers
}

\author{
Pavani Rangachari \\ Department of Interdisciplinary Health Sciences and Department of Family Medicine, Augusta University, Augusta, GA, USA \\ Correspondence to: Pavani Rangachari, PhD, CPH. Department of Interdisciplinary Health Sciences and Department of Family Medicine, Augusta \\ University, Augusta, GA 30912, USA. Email: PRANGACHARI@augusta.edu. \\ Comment on: Petry NJ, Van Heukelom J, Baye JF, et al. The effect of medication reconciliation on generating an accurate medication list in a \\ pharmacogenomics practice. Pharmacogenomics Res Pers Med 2021. doi: 10.21037/prpm-21-7.
}

Received: 05 December 2021; Accepted: 21 December 2021.

doi: 10.21037/prpm-21-25

View this article at: https://dx.doi.org/10.21037/prpm-21-25

\section{Introduction}

Recent efforts to reform the healthcare delivery system have focused on improving patient safety during transitions of care, when patients move between healthcare providers and settings as their conditions change during the course of an illness (1-5). Medication errors, which represent the most common patient safety error during care transitions (e.g., admission, transfer, and discharge), are estimated to harm 1.5 million people and cost at least $\$ 3.5$ billion per year. Adverse drug events are also estimated to affect approximately 2 million hospital stays per year and prolong the length of stay by 1.7-4.6 days (6,7). Transitions of care pose a higher risk for medication errors due to greater potential for communication breakdowns regarding patient medications between healthcare settings. Over 40 percent of medication errors are known to result from inadequate reconciliation of patient medications during transitions of care. In other words, many of these errors could be averted if consistent medication reconciliation (MedRec) processes were in place (5-7).

\section{Federal policy emphasizes "meaningful use" of electronic health record (EHR) MedRec}

MedRec is the formal process for creating the most complete and accurate list of a patient's current medications. It involves: (I) developing a list of the patient's current medications; (II) developing a list of medications to be prescribed; (III) comparing both medication lists; (IV) making clinical decisions to continue, discontinue, or add new medications based on the comparison; and (V) communicating the final, updated list to the patient, caregivers, and the next level of providers (6).

Since 2007, MedRec has been part of the Joint Commission's National Patient Safety Goals Program (1). In 2011, MedRec became part of the US Centers for Medicare and Medicaid Services (CMS) EHR "meaningful use" requirements. Meaningful use refers to the utilization of a certified EHR system to improve quality, safety, efficiency, care coordination, and population health, engage patients and families in their own health care, and reduce health and healthcare disparities $(8,9)$. Healthcare providers and organizations could receive incentives for meeting metrics for meaningful use of EHR MedRec defined by CMS as "the process of identifying the most accurate list of all medications that the patient is taking, including name, dosage, frequency, and route, by comparing the medical record to an external list of medications obtained from a patient, hospital, or other provider" $(8,9)$.

\section{Healthcare organizations suffer from "limited use" of EHR MedRec}

Despite the emphasis on meaningful use of EHR MedRec at a federal policy level, the implementation of EHR MedRec at the healthcare provider or organizational level, is known to be limited or inconsistent. A key barrier has been 
identified as low physician engagement, resulting in part from a lack of professional consensus on who is responsible for managing the patient's medication list (e.g., hospital physician $v s$. clinic/community physician) $(4,10)$. In general, physicians are also known to be resistant to discontinuing a medication (on the list), that they did not originally order. Additionally, within the context of the hospital organization, the division of roles and responsibilities related to EHR MedRec across various practitioner groups, i.e., multiple physicians, pharmacists, and nurses, is often unclear, leading to greater potential for medication errors (10-13). Many recent studies that have echoed these concerns, have found that despite continuous improvements to the MedRec functionality on EHR systems over time, many hospitals continue to use partially paper-based processes during transitions of care $(11,14)$. In other words, there is a tendency on the part of healthcare providers to "work around" the EHR system and communicate changes in medication lists directly to the patient or caregiver, resulting in "limited use" of the EHR MedRec functionality, as opposed to "meaningful use".

\section{Challenges with EHR MedRec from the healthcare provider perspective}

Studies that have examined challenges in EHR MedRec from the provider perspective, have identified various types of issues, including care coordination issues, patient education issues, ownership issues, process issues, information technology issues, training issues, workflow misalignment issues, resource issues, and documentation issues $(4,10,12,14-16)$. Care coordination issues may result from (I) lack of arrangements for post-discharge follow-up to monitor interactions of new medicines with the patient's existing medications, or from (II) pharmacists not being able to meet patients at the time of discharge to educate patients and reconcile medications before they leave the hospital and return to the community. On the hand, patient education issues may arise from patients not understanding when one medication on their list is being replaced by another (e.g., in the case of beta-blockers). These types of patient comprehension issues in turn, could prevent healthcare providers from being able to compile a complete and accurate medication list at the time of patient arrival (which in turn, could affect the accuracy of the medication list upon discharge).

Ownership issues may result from an absence of consensus regarding which provider is responsible for reconciling or discontinuing drugs on the medication list. For example, a beta-blocker on hospital formulary may have been ordered to replace the beta-blocker that the patient normally takes at home, however, the former drug may not have been discontinued at discharge. The presence of both beta-blockers on the discharge medication list in turn, creates potential for an adverse outcome through drug duplication. Even within the hospital setting, the absence of clarity related to roles \& responsibilities, may prompt multiple practitioners (nurses, pharmacists, physicians) to check-off that they each completed the MedRec process. However, in the absence of ownership, nobody could be held accountable for ensuring a complete and accurate medication list, a problem that could simultaneously result in multiple "active" lists within the EHR system and dissatisfied patients (from being questioned about their medications by multiple providers during the course of a single hospital stay). Process of care issues may result from MedRec not being performed during the course of hospital stay (to update the medication list developed at admission based on new information obtained from family members), which in turn, has potential to affect the accuracy of the discharge medication list.

Information technology-related issues may arise from lack of EHR interoperability across hospitals within the local community, while workforce training issues may arise from all providers not fully understanding the importance of continuously updating the medication list for ensuring patient safety after discharge and preventing readmissions resulting from medication errors. Workflow issues may occur if the medication history is not recorded comprehensively in a busy clinic, leading to inaccurate medication information being inputted into the patient's EHR. Likewise, resource issues may occur if there is a shortage of staff to assist with MedRec during discharge, while documentation issues may occur if the medication list is lacking essential details related to medication names, types, routes, and dosages.

\section{Broader issue of "absence of shared understanding" related to EHR MedRec among providers}

A key insight from the above discussion on challenges encountered by practitioners in context of EHR MedRec, is that these challenges are reflective of a broader underlying problem-the absence of shared understanding across multiple provider subgroups in regard to: (I) why EHR 
MedRec is necessary, i.e., its value in preventing errors and promoting safety; (II) what the responsibilities are of each provider subgroup in the EHR MedRec process; (III) how the EHR MedRec system is being used by other provider subgroups (15).

\section{Need for shared understanding of the value of EHR MedRec to promote meaningful use}

Insights gained from the discussion on challenges experienced by providers in the context of EHR MedRec, suggest that meaningful use of EHR MedRec requires a foundation of inter-professional learning and shared understanding of the value of EHR MedRec in preventing errors and promoting patient safety. According to the "professional complex systems" framework, a knowledge sharing network in which senior leaders within a healthcare organization, play a proactive role in promoting "tacit knowledge exchange" across professional subgroups, could be effective in enabling collective (inter-professional) learning and practice change (e.g., EHR meaningful use) (17-20). In the context of EHR MedRec, an example of tacit knowledge exchange would be a community nurse sharing with a hospital physician how a clinic patient ended up being readmitted to the hospital, as a result of an overdose of beta-blocker medication (which in turn, resulted from not discontinuing the hospital formulary beta-blocker on the patient medication list at discharge when a home version was present). The exchange of tacit knowledge among healthcare providers in turn, has the potential to: (I) establish a direct connection between gaps in (EHR MedRec) practices and adverse patient outcomes; and thereby (II) underscore the importance of following best practices in EHR MedRec. Understanding the direct linkages between provider practices and their consequences for patients, could help to increase provider engagement in addressing issues related to EHR MedRec, which in turn, could provide a foundation for providers to learn collectively, and change their practices, ultimately leading to more meaningful use of the MedRec functionality on the EHR.

\section{Transitioning from "limited use" to "meaningful use" of EHR MedRec}

In recent years, health services researchers have drawn upon the "professional complex systems" framework to design systems for inter-professional learning related to EHR MedRec best practices (and shared understanding of the value of EHR MedRec for patient safety), to enable transition from "limited use" of EHR MedRec technology to "meaningful use". In one academic health center, a leader-moderated knowledge sharing system related to EHR MedRec was developed to enable practitioners to engage in tacit knowledge exchange on issues related to EHR MedRec and benefit from collective learning of best practices. The latter in turn, helped to develop shared understanding of the link between EHR MedRec practices and patient outcomes, which in turn paved the way for more meaningful use of the EHR MedRec functionality (21-24).

For example, the knowledge sharing system developed at this institution, helped to facilitate tacit knowledge exchange and collective inter-professional learning with respect to the EHR MedRec best-practice of "consistent importation of the external Rx history into the EHR system at the start of patient encounters". This best-practice in turn, corresponds to a broader set of best-practices targeted towards improving communication across the continuum of healthcare providers regarding the patient's medication list. "External Rx history import" in turn, refers to providers' practice of importing the history of medications filled at the patient's pharmacy, early in the patient encounter, by activating the Rx history command on the EHR system.

To elaborate on the process, inter-professional knowledge exchange related to "external Rx history import" began with a problem statement from a nurse in the emergency department (ED), that it was challenging to obtain an accurate medication history upon a patient's arrival in the $\mathrm{ED}$, to compile an accurate active medication list. Another nurse from the ambulatory setting replied suggesting use of the "external Rx history import," functionality within the EHR, to obtain the current medication list from the patient's pharmacy. There was however, some pushback to this suggestion from both the original emergency nurse who argued that the list generated by the external Rx function could not be trusted and a cardiologist (physician) who argued that relying solely on this functionality in the absence of physical pill bottles from the patient would provide unreliable and incomplete information. There were however, others who responded to the push-back with problem-solving statements. For example, a hospitalist argued that even if the list from external Rx may not be comprehensive, it could provide a starting point for discussing the active medication list with the patient or caregiver. Another Medical Informatics physician leader then responded with IT system education related to the external Rx history option on the EHR. 




Figure 1 Evidence-based strategies for enabling meaningful use of EHR MedRec technology. EHR, electronic health record; MedRec, Medication reconciliation.

There were also best-practice assertions highlighting the fact that performing external Rx history import would at the very least enable providers to obtain an initial list of prescriptions to begin a discussion regarding what the patient was actually taking.

One of the leader moderators then helped to reinforce these best-practice assertions, by providing real-time examples of how timely use of the external Rx history option may have helped to avert dosing errors resulting from verbal orders. At this juncture, a pharmacist made a culture change assertion, urging physician colleagues to not allow "the ideal to interfere with the good or the better". In this case, the argument from the pharmacist, was that when prescribers fail to reconcile medications by claiming insufficient information, in effect, they are deciding to "do nothing". These discussions then culminated in a collective learning (a-ha) moment summarized by the leader-moderators of the knowledge sharing system, i.e., that leveraging the external Rx history import function to incrementally improve the accuracy of the patient's active medication list, could go a long way in helping to reduce medication errors during transitions of care.

The aforementioned dynamics of inter-professional knowledge exchange and collective learning in turn, coincided with a distinct improvement trend in the proportion of external Rx history import among patients undergoing treatment for congestive heart failure within the health system, and specifically, within the "medicine service lines" that participated in the knowledge sharing system established at the institution. This in turn, helped to infer these systematic efforts from health system leadership, to promote inter-professional learning related to EHR MedRec best practices and shared understanding of the value of those best practices in preventing errors, which can in fact help to promote meaningful use of EHR MedRec technology.

\section{Evidence-based management strategies for meaningful use of EHR MedRec}

The body of research discussed earlier, has helped to identify evidence-based management strategies for inter-professional learning and change in the context of meaningful use of EHR MedRec. These strategies are summarized in Figure 1 (15,21-24).

An article featured in this issue by Petry et al. entitled "Medication reconciliation and pharmacogenetic reviews: the importance of an accurate medication list" helps to reinforce the value of these evidence-based management strategies by describing how institutional efforts to 
improve the EHR MedRec workflow, could go a long way in improving medication list accuracy (and preventing medication errors) (25). The article describes how the introduction of a dedicated team of student pharmacists in the EHR MedRec workflow within a precision medicine preemptive screening program at an institution, helped to significantly improve the accuracy of patient medication lists at admission and discharge. Results of the intervention served to highlight the value of having a dedicated team of student pharmacists for MedRec in precision medicine, which in turn, can serve to promote shared understanding among providers, of the value of accurate medication lists on the EHR, in preventing medication errors and optimizing patient outcomes in precision medicine.

\section{Conclusions}

This article helped to understand how healthcare organizations could strive to create shared understanding of the value of EHR MedRec across multiple professional subgroups, by developing systems for knowledge sharing and collective learning related to best practices in EHR MedRec, and by highlighting the potential of these practices to prevent errors and promote safety. The discussion of lessons learned from this body of research in turn, helps to reinforce previously identified changemanagement strategies for enabling meaningful use of the MedRec functionality within EHR systems in healthcare organizations.

\section{Acknowledgments}

Funding: The studies described in this Editorial Article were supported by grant number R21HS024335 from the Agency for Healthcare Research and Quality.

\section{Footnote}

Provenance and Peer Review: This article was commissioned by the editorial office, Pharmacogenomics Research and Personalized Medicine. The article did not undergo external peer review.

Conflicts of Interest: The author has completed the ICMJE uniform disclosure form (available at https://dx.doi. org/10.21037/prpm-21-25). The authors have no conflicts of interest to declare.
Ethical Statement: The author is accountable for all aspects of the work in ensuring that questions related to the accuracy or integrity of any part of the work are appropriately investigated and resolved.

Open Access Statement: This is an Open Access article distributed in accordance with the Creative Commons Attribution-NonCommercial-NoDerivs 4.0 International License (CC BY-NC-ND 4.0), which permits the noncommercial replication and distribution of the article with the strict proviso that no changes or edits are made and the original work is properly cited (including links to both the formal publication through the relevant DOI and the license). See: https://creativecommons.org/licenses/by-nc-nd/4.0/.

\section{References}

1. Burton, R. Health policy brief: improving care transitions. Health Affairs 2012. Available online: http:// www.healthaffairs.org/healthpolicybriefs/brief.php?brief_ id $=76$

2. Coleman EA. Falling through the cracks: challenges and opportunities for improving transitional care for persons with continuous complex care needs. J Am Geriatr Soc 2003;51:549-55.

3. AHRQ, Agency for Healthcare Research and Quality (2016). Patient safety primer: medication reconciliation. Available online: https://psnet.ahrq.gov/primers/primer/1/ medication-reconciliation. Accessed October 15, 2016.

4. Mueller SK, Sponsler KC, Kripalani S, et al. Hospitalbased medication reconciliation practices: a systematic review. Arch Intern Med 2012;172:1057-69.

5. Aspden P, Wolcott JA, Bootman JL, et al. Preventing medication errors: quality chasm series. Washington, DC: National Acad Press, 2007.

6. Barnsteiner, JH. Medication Reconciliation. In: Hughes RG, editor. Patient Safety and Quality: An EvidenceBased Handbook for Clinicians. Rockville (MD): Agency for Healthcare Research and Quality (US); 2008:Chapter 38. Available from: http://www.ncbi.nlm.nih.gov/books/ NBK2648/

7. da Silva BA, Krishnamurthy M. The alarming reality of medication error: a patient case and review of pennsylvania and national data. J Community Hosp Intern Med Perspect 2016;6:31758.

8. CMS centers for medicare and medicaid services (2012). Stage 2 eligible professional meaningful use core 
measures measure 14 of 17 . Available online: https:// www.cms.gov/Regulations-and-Guidance/Legislation/ EHRIncentivePrograms/downloads/Stage2_EPCore_14_ MedicationReconciliation.pdf

9. HealthIT.Gov. (2016). Achieving Meaningful Use Stage 2: Medication Reconciliation. Available online: https://www. healthit.gov/providers-professionals/achieve-meaningfuluse/core-measures-2/medication-reconciliation

10. Boockvar KS, Santos SL, Kushniruk A, et al. Medication reconciliation: barriers and facilitators from the perspectives of resident physicians and pharmacists. J Hosp Med 2011;6:329-37.

11. Grossman JM, Gourevitch, R, Cross DA. Hospital experiences using electronic health records to support medication reconciliation. National Institute for Health Care Reform (NIHCR) Research Brief Number 17, 2014.

12. Sanchez SH, Sethi SS, Santos SL, Boockvar, K. Implementing medication reconciliation from the planner's perspective: a qualitative study. BMC Health Services Research 2014;14:290.

13. van Sluisveld N, Zegers M, Natsch S, et al. Medication reconciliation at hospital admission and discharge: insufficient knowledge, unclear task reallocation and lack of collaboration as major barriers to medication safety. BMC Health Serv Res 2012;12:170.

14. Pevnick JM, Shane R, Schnipper JL. The problem with medication reconciliation. BMJ Qual Saf 2016;25:726-30.

15. Rangachari P, Dellsperger KC, Fallaw D, et al. A mixedmethod study of practitioners' perspectives on issues related to ehr medication reconciliation at a health system. Qual Manag Health Care 2019;28:84-95.

16. Porcelli PJ, Waitman LR, Brown SH. A review of medication reconciliation issues and experiences with clinical staff and information systems. Appl Clin Inform 2010;1:442-61.

17. Rangachari P. Knowledge sharing networks related to hospital quality measurement and reporting. Health Care Manage Rev 2008;33:253-63.

doi: $10.21037 /$ prpm-21-25

Cite this article as: Rangachari P. Meaningful use of electronic health record (EHR) medication reconciliation technology requires a shared understanding of its value among healthcare providers. Pharmacogenomics Res Per Med 2021.
18. Rangachari P. Overcoming the Unintended Adverse Consequences of Implementing Health IT through Human Resource and Knowledge Management. In: Fottler MD, Khatri N, Savage GT, editors. Strategic Human Resource Management in Health Care, Advances in Health Care Management, Bingley, UK: Emerald, 2010:163-94.

19. Rangachari P, Madaio M, Rethemeyer RK, et al. The evolution of knowledge exchanges enabling successful practice change in two intensive care units. Health Care Manage Rev 2015;40:65-78.

20. Rangachari P. Implementing a social knowledge networking (SKN) system to enable meaningful use of an EHR medication reconciliation system. Risk Manag Healthc Policy 2018;11:45-53.

21. Rangachari P. Innovation implementation in the context of hospital QI: lessons learned and strategies for success. Innov Entrep Health 2018;5:1-14.

22. Rangachari P, Dellsperger KC, Rethemeyer RK. A qualitative study of interprofessional learning related to electronic health record (EHR) medication reconciliation within a social knowledge networking (SKN) system. J Healthc Leadersh 2019;11:23-41.

23. Rangachari P, Dellsperger KC, Rethemeyer RK. Network analysis of the structure of inter-professional knowledge exchange related to electronic health record medication reconciliation within a social knowledge networking system. J Healthc Leadersh 2019;11:87-100.

24. Rangachari P, Dellsperger KC, Rethemeyer RK. A health system's pilot experience with using mobile social knowledge networking (SKN) technology to enable meaningful use of EHR medication reconciliation technology. AMIA Annu Symp Proc 2019;2019:745-54.

25. Petry NJ, Van Heukelom J, Baye JF, et al. The effect of medication reconciliation on generating an accurate medication list in a pharmacogenomics practice. Pharmacogenomics Res Pers Med 2021. doi: 10.21037/ prpm-21-7. 